\title{
Semilinear degenerate elliptic boundary value problems at resonance
}

\author{
Kazuaki Taira \\ Dedicated to Professor Seiichiro Wakabayashi on the occasion of his 60th birthday
}

Received: date / Accepted: date

\begin{abstract}
The purpose of this paper is to study a class of semilinear degenerate elliptic boundary value problems at resonance which include as particular cases the Dirichlet and Robin problems. The approach here is based on the global inversion theorems between Banach spaces, and is distinguished by the extensive use of the ideas and techniques characteristic of the recent developments in the theory of partial differential equations. By making use of the Lyapunov-Schmidt procedure and the global inversion theorem, we prove existence and uniqueness theorems for our problem. The results here extend an earlier theorem due to Landesman and Lazer to the degenerate case.
\end{abstract}

Keywords Semilinear boundary value problem at resonance · degenerate boundary condition · global inversion theorem · the Lyapunov-Schmidt procedure

Mathematics Subject Classification (2000) 35J65 - 35J25 · 47H10

\section{Statement of main results}

Let $\Omega$ be a bounded domain of Euclidean space $\mathbf{R}^{N}, N \geq 2$, with smooth boundary $\partial \Omega$; its closure $\bar{\Omega}=\Omega \cup \partial \Omega$ is an $N$-dimensional, compact smooth manifold with boundary. Let $A$ be a second-order, elliptic differential operator with real coefficients such that

$$
A u=-\sum_{i=1}^{N} \frac{\partial}{\partial x_{i}}\left(\sum_{j=1}^{N} a^{i j}(x) \frac{\partial u}{\partial x_{j}}\right)+c(x) u .
$$

Here:

(1) $a^{i j} \in C^{\infty}(\bar{\Omega})$ and $a^{i j}(x)=a^{j i}(x)$ for all $x \in \bar{\Omega}$ and $1 \leq i, j \leq N$, and there exists a positive constant $a_{0}$ such that

$$
\sum_{i, j=1}^{N} a^{i j}(x) \xi_{i} \xi_{j} \geq a_{0}|\xi|^{2} \quad \text { for all }(x, \xi) \in \bar{\Omega} \times \mathbf{R}^{N} .
$$

Kazuaki Taira

Institute of Mathematics, University of Tsukuba, Tsukuba 305-8571, Japan

E-mail: taira@math.tsukuba.ac.jp 
(2) $c \in C^{\infty}(\bar{\Omega})$ and $c(x) \geq 0$ in $\Omega$.

Let $B$ be a first-order, boundary condition with real coefficients such that

$$
B u=a\left(x^{\prime}\right) \frac{\partial u}{\partial \boldsymbol{\nu}}+b\left(x^{\prime}\right) u
$$

Here:

(3) $a \in C^{\infty}(\partial \Omega)$ and $a\left(x^{\prime}\right) \geq 0$ on $\partial \Omega$.

(4) $b \in C^{\infty}(\partial \Omega)$ and $b\left(x^{\prime}\right) \geq 0$ on $\partial \Omega$.

(5) $\partial / \partial \boldsymbol{\nu}$ is the conormal derivative associated with the operator $A$ :

$$
\frac{\partial}{\partial \boldsymbol{\nu}}=\sum_{i, j=1}^{N} a^{i j}\left(x^{\prime}\right) n_{j} \frac{\partial}{\partial x_{i}}
$$

where $\mathbf{n}=\left(n_{1}, n_{2}, \ldots, n_{N}\right)$ is the unit exterior normal to the boundary $\partial \Omega$.

Our fundamental hypotheses on the boundary condition $B$ are the following:

(H.1) $a\left(x^{\prime}\right)+b\left(x^{\prime}\right)>0$ on $\partial \Omega$.

(H.2) $b\left(x^{\prime}\right) \not \equiv 0$ on $\partial \Omega$.

The intuitive meaning of hypotheses (H.1) and (H.2) is that either the reflection phenomenon or the absorption phenomenon does occur at each point of the boundary $\partial \Omega$. More precisely, condition (H.1) implies that the absorption phenomenon occurs at each point of the set $M=\left\{x^{\prime} \in \partial \Omega: a\left(x^{\prime}\right)=0\right\}$, while the reflection phenomenon occurs at each point of the set $\partial \Omega \backslash M=\left\{x^{\prime} \in \partial \Omega: a\left(x^{\prime}\right)>0\right\}$. In other words, a Markovian particle moves continuously in the space $\bar{\Omega} \backslash M$ until it dies at the time when it reaches the set $M$ where the particle is definitely absorbed (see [15]). On the other hand, condition (H.2) implies that the boundary condition $B$ is not equal to the purely Neumann condition. We remark that if $a\left(x^{\prime}\right) \equiv 0$ and $b\left(x^{\prime}\right) \equiv 1$ on $\partial \Omega$ (resp. $a\left(x^{\prime}\right)>0$ on $\partial \Omega$ ), then the boundary condition $B$ is the Dirichlet condition (resp. Robin condition).

It is easy to see that $B$ is non-degenerate (or coercive) if and only if either $a\left(x^{\prime}\right)>0$ on $\partial \Omega$ or $a\left(x^{\prime}\right) \equiv 0$ and $b\left(x^{\prime}\right)>0$ on $\partial \Omega$. In other words, $B$ is a degenerate boundary condition from an analytical point of view. This is due to the fact that the so-called Shapiro-Lopatinskii complementary condition is violated at each point of the set $M$ (cf. [7]). Amann [2] studied the case where the boundary $\partial \Omega$ is the disjoint union of the two closed subsets $M$ and $\partial \Omega \backslash M$, each of which is an $(N-1)$ dimensional compact smooth manifold.

In this paper we consider the following semilinear elliptic boundary value problem at resonance: Let $q(\xi)$ be a function defined on $\mathbf{R}$. Given a function $h(x)$ in $\Omega$, find a function $u(x)$ in $\Omega$ such that

$$
\begin{cases}-A u+\lambda_{1} u+q(u)=h & \text { in } \Omega, \\ B u=a\left(x^{\prime}\right) \frac{\partial u}{\partial \boldsymbol{\nu}}+b\left(x^{\prime}\right) u=0 & \text { on } \partial \Omega .\end{cases}
$$

It should be emphasized that the linear part $-A+\lambda_{1} I$ is not invertible and further that problem (1.3) may have no solution at all. Indeed, this is the case where $q(\xi) \equiv 0$ on $\mathbf{R}$, that is, the linear case. The purpose of this paper is to prove existence and uniqueness theorems for problem (1.3) in the framework of Hölder spaces. We remark that existence 
and uniqueness theorems for problem (1.3) were first obtained by Landesman-Lazer [9] in the framework of Sobolev spaces of $L^{2}$ type (see also [13, Section 6.4]).

In order to study problem (1.3) in the framework of Hölder spaces, we consider the linear elliptic boundary value problem

$$
\begin{cases}A u=g & \text { in } \Omega, \\ B u=0 & \text { on } \partial \Omega\end{cases}
$$

in the framework of the Hilbert space $L^{2}(\Omega)$. We associate with problem (1.4) a densely defined, closed linear operator

$$
\mathfrak{A}: L^{2}(\Omega) \longrightarrow L^{2}(\Omega)
$$

as follows:

(a) $D(\mathfrak{A})=\left\{u \in W^{2,2}(\Omega): B u=0\right.$ on $\left.\partial \Omega\right\}$.

(b) $\mathfrak{A} u=A u$ for all $u \in D(\mathfrak{A})$.

Here and in the following the Sobolev space $W^{k, p}(\Omega)$ for $k \in \mathbf{N}$ and $1<p<\infty$ is defined as follows:

$$
\begin{aligned}
W^{k, p}(\Omega)= & \text { the space of functions } u \in L^{p}(\Omega) \text { whose derivatives } D^{\alpha} u, \\
& |\alpha| \leq k, \text { in the sense of distributions are in } L^{p}(\Omega),
\end{aligned}
$$

and its norm $\|\cdot\|_{W^{k, p}(\Omega)}$ is given by the formula

$$
\|u\|_{W^{k, p}(\Omega)}=\left(\sum_{|\alpha| \leq k} \int_{\Omega}\left|D^{\alpha} u(x)\right|^{p} d x\right)^{1 / p}
$$

Then we have the following fundamental spectral results (i), (ii) and (iii) of the operator $\mathfrak{A}$ (see [16, Theorem 5.1]):

(i) The operator $\mathfrak{A}$ is positive and selfadjoint in $L^{2}(\Omega)$.

(ii) The first eigenvalue $\lambda_{1}$ of $\mathfrak{A}$ is positive and algebraically simple, and its corresponding eigenfunction $\phi_{1} \in C^{2+\alpha}(\bar{\Omega})$, with exponent $0<\alpha<1$, may be chosen to be strictly positive in $\Omega$. Namely, we have the assertions

$$
\begin{cases}A \phi_{1}=\lambda_{1} \phi_{1} & \text { in } \Omega, \\ \phi_{1}>0 & \text { in } \Omega, \\ B \phi_{1}=0 & \text { on } \partial \Omega .\end{cases}
$$

(iii) No other eigenvalues $\lambda_{j}, j \geq 2$, have positive eigenfunctions.

Now we impose the following three conditions (B.1), (B.2) and (B.3) on the nonlinear term $q(\xi)$ :

(B.1) The function $q(\xi)$ is real-valued and bounded on $\mathbf{R}$.

(B.2) The function $q(\xi)$ is of class $C^{1+\alpha}$ with $0<\alpha<1$ on $\mathbf{R}$ and satisfies the condition

$$
q^{\prime}(\xi)<\lambda_{2}-\lambda_{1} \quad \text { for all } \xi \in \mathbf{R}
$$

where $\lambda_{1}$ and $\lambda_{2}$ are eigenvalues of $\mathfrak{A}$.

(B.3) The finite limits $q^{+}=\lim _{\xi \rightarrow+\infty} q(\xi)$ and $q^{-}=\lim _{\xi \rightarrow-\infty} q(\xi)$ exist and $q^{-}<q^{+}$. 
Example 1.1 A simple example of the nonlinear term $q(\xi)$ is given by the formula

$$
q(\xi)= \begin{cases}\gamma\left(-\frac{1}{2 \xi}+\frac{3}{4}\right) & \text { for } \xi>1 \\ \frac{\gamma}{4} \xi^{2} & \text { for } 0 \leq \xi \leq 1 \\ -\frac{\delta}{4} \xi^{2} & \text { for }-1 \leq \xi<0 \\ \delta\left(-\frac{1}{2 \xi}-\frac{3}{4}\right) & \text { for } \xi<-1\end{cases}
$$

Here $0<\gamma<2\left(\lambda_{2}-\lambda_{1}\right)$ and $0<\delta<2\left(\lambda_{2}-\lambda_{1}\right)$. It is easy to see that this function $q(\xi)$ satisfies condition (B.3) with $q^{+}:=3 \gamma / 4$ and $q^{-}:=-3 \delta / 4$.

The next existence theorem is a generalization of Ambrosetti-Prodi [3, Chapter 4, Theorem 1.8] to the degenerate case (cf. [9, Theorem]):

Theorem 1.1 Assume that the nonlinear term $q(\xi)$ satisfies conditions (B.1), (B.2) and (B.3). If the function $h \in C^{\alpha}(\bar{\Omega})$ satisfies the condition

$$
q^{-} \int_{\Omega} \phi_{1}(x) d x<\int_{\Omega} h(x) \phi_{1}(x) d x<q^{+} \int_{\Omega} \phi_{1}(x) d x
$$

then problem (1.3) has a solution $u \in C^{2+\alpha}(\bar{\Omega})$.

Now we consider the case where condition (B.3) is violated. To do this, we introduce (replacing condition (B.3)) the following condition (B.4) on the nonlinear term $q(\xi)$ :

(B.4) The finite limit $\sigma=\lim _{|\xi| \rightarrow \infty} \xi q(\xi)$ exists and $\sigma>0$.

Example 1.2 A simple example of the nonlinear term $q(\xi)$ is given by the formula

$$
q(\xi)=\left(\lambda_{2}-\lambda_{1}\right) \frac{\xi}{1+\xi^{2}} \quad \text { for all } \xi \in \mathbf{R} .
$$

This function $q(\xi)$ satisfies condition (B.4) with $\sigma:=\lambda_{2}-\lambda_{1}$.

The next existence theorem is a generalization of Ambrosetti-Prodi [3, Chapter 4, Theorem 1.10] to the degenerate case (cf. [9, Theorem]):

Theorem 1.2 Assume that the nonlinear term $q(\xi)$ satisfies conditions (B.1), (B.2) and (B.4). If the function $h \in C^{\alpha}(\bar{\Omega})$ satisfies the orthogonal condition

$$
\int_{\Omega} h(x) \phi_{1}(x) d x=0
$$

then problem (1.3) has a solution $u \in C^{2+\alpha}(\bar{\Omega})$.

Remark 1.1 By arguing as in the proof of Theorem 1.2 (see Section 5), we can prove that there exists a positive constant $\varepsilon$ such that problem (1.3) has at least two solutions $u_{1}, u_{2} \in C^{2+\alpha}(\bar{\Omega})$ if the function $h \in C^{\alpha}(\bar{\Omega})$ satisfies the condition

$$
\left|\int_{\Omega} h(x) \phi_{1}(x) d x\right|<\varepsilon
$$

Finally, the next existence and uniqueness theorem is a generalization of Ambrosetti-Prodi [3, Chapter 4, Theorem 1.12] to the degenerate case (cf. [9, Theorem]): 
Theorem 1.3 Assume that the nonlinear term $q(\xi)$ satisfies conditions (B.1), (B.2) and (B.3), and further that $q^{\prime}(\xi)>0$ on $\mathbf{R}$. Then problem (1.3) has a unique solution $u \in C^{2+\alpha}(\bar{\Omega})$ if and only if the function $h \in C^{\alpha}(\bar{\Omega})$ satisfies condition (1.6).

The rest of this paper is organized as follows. Section 2 deals with local and global inversions of mappings between Banach spaces which go back to Hadamard in the finite dimensional case and to Cacciopoli and Lévy for general Banach spaces. Our proof of Theorems 1.1, 1.2 and 1.3 is based on the Lyapunov-Schmidt procedure which reduces an infinite-dimensional problem to a finite-dimensional system. Section 3 is devoted to the Lyapunov-Schmidt procedure (Proposition 3.1). This section is the heart of the subject, and is based on the previous work [16] and [17]. More precisely, we make use of a generalization of the classical Krein-Rutman theory ([8]) to the degenerate case ([16]) and also a generalization of the classical variational approach ([5]) to eigenvalue problems with an indefinite weight to the degenerate case ([17]). By virtue of the global inversion theorem (Theorem 2.3), we are reduced to the study of a one-dimensional system (equation (3.5)). In Section 4 we study equation (3.5), and prove Theorem 1.1, by using the intermediate value theorem. Similarly, Theorem 1.2 and Theorem 1.3 are proved in Section 5 and Section 6, respectively.

\section{Local and global inversion theorems}

This section deals with local and global inversions of mappings between Banach spaces which go back to Hadamard in the finite dimensional case and to Cacciopoli and Lévy for general Banach spaces (Theorem 2.3). The presentation here is taken from Ambrosetti-Prodi [3], Dieudonné [6] and Nirenberg [12] (see also [4], [11], [18]).

\subsection{Local inversion theorem}

Let $X$ and $Y$ be Banach spaces and let $F: X \rightarrow Y$ be a $C^{1}$ map. Namely, the map $F$ is differentiable in $X$ and the Fréchet derivative $D F$ is continuous as a map of $X$ into the space $B(X, Y)$ of bounded (continuous) linear operators on $X$ into $Y$.

A continuous map $F: X \rightarrow Y$ is said to be locally invertible at a point $u^{*}$ of $X$ if there exist an open neighborhood $U$ of $u^{*}$, an open neighborhood $V$ of $F\left(u^{*}\right)$ and a continuous map $G: V \rightarrow U$ such that

$$
\begin{cases}G(F(u))=u & \text { for all } u \in U, \\ F(G(v))=v & \text { for all } v \in V .\end{cases}
$$

The map $G$ is called the local inverse of $F$, and will be denoted by $F^{-1}$.

The next local inversion theorem provides a criterion for a map to be a local $C^{1}$ diffeomorphism in terms of its Fréchet derivative (see [6, Theorem 10.2.5]; [3, Chapter 2, Theorem 1.2]):

Theorem 2.1 Let $F$ be a $C^{1}$ map of $X$ into $Y$. Assume that the Fréchet derivative $D F\left(u^{*}\right): X \rightarrow Y$ is continuous and invertible at a point $u^{*} \in X$. Then $F$ is locally invertible at $u^{*}$ with $C^{1}$ inverse $F^{-1}$. More precisely, there exist an open neighborhood $U$ of $u^{*}$ and an open neighborhood $V$ of $F\left(u^{*}\right)$ such that the inverse $F^{-1}: V \rightarrow U$ is $a C^{1}$ map and that $D\left(F^{-1}\right)(v)=(D F(u))^{-1}$ for all $v=F(u)$ with $u \in U$. 
The process of linearization provides a key link between the linear and nonlinear theories of partial differential equations. Our basic tool is the following implicit function theorem that is one of the most important applications of Theorem 2.1 (see [6, Theorem 10.2.1]; [12, Theorem 2.7.5]):

Theorem 2.2 Let $X, Y, Z$ be Banach spaces, and let $f$ be a $C^{1}$-map of an open subset $U \times V$ of $X \times Y$ into $Z$. Assume that the Fréchet partial derivative $D_{y} f\left(x_{0}, y_{0}\right): Y \rightarrow Z$ is an algebraic and topological isomorphism at a point $\left(x_{0}, y_{0}\right)$ of $U \times V$. Then there exist neighborhoods $U_{0}$ of $x_{0}$ and $W_{0}$ of $f\left(x_{0}, y_{0}\right)$ and a unique $C^{1} \operatorname{map} g: U_{0} \times W_{0} \rightarrow V$ such that $f(x, g(x, w))=w$ for all $(x, w) \in U_{0} \times W_{0}$.

\subsection{Global inversion theorem}

Let $M$ and $N$ be metric spaces and let $F: M \rightarrow N$ be a continuous map. The map $F: M \rightarrow N$ is said to be proper if the preimage $F^{-1}(K)$ is compact in $M$ for any compact set $K$ in $N$. We remark that if $F$ is proper, then it maps closed sets in $M$ into closed sets in $N$.

A topological space $T$ is said to be simply connected if it is arcwise connected and if every closed path $\sigma$ in $T$ is homotopic to a constant. Namely, for any given map $\sigma \in C([0,1], T)$ with $\sigma(0)=\sigma(1)$ there exist a map $h \in C([0,1] \times[0,1], T)$ and a point $v \in T$ such that

$$
\begin{cases}h(s, 0)=\sigma(s) & \text { for } 0 \leq s \leq 1 \\ h(s, 1)=v & \text { for } 0 \leq s \leq 1 \\ h(0, t)=h(1, t) & \text { for } 0 \leq t \leq 1\end{cases}
$$

Now we are in position to state the global inversion theorem (see [3], Chapter 3, Theorem 1.8):

Theorem 2.3 Let $M$ be an arcwise connected metric space and let $N$ be a simply connected metric space. Assume that a continuous map $F: M \rightarrow N$ is proper and locally invertible on all of $M$. Then the map $F$ is a homeomorphism of $M$ onto $N$.

\section{Lyapunov-Schmidt procedure}

The main idea of this section is to rewrite problem (1.3) in a suitable bifurcation system (the Lyapunov-Schmidt procedure) and to formulate conditions which imply a priori estimates for solutions of problem (1.3) (see Proposition 3.1). This section is divided into two subsections.

\subsection{Orthogonal decomposition in Hölder spaces}

First, we have the following orthogonal decomposition in the Hilbert space $L^{2}(\Omega)$ :

$$
\begin{aligned}
L^{2}(\Omega)= & N\left(\mathfrak{A}-\lambda_{1} I\right) \oplus R\left(\mathfrak{A}-\lambda_{1} I\right) \\
= & \left\{u \in L^{2}(\Omega):\left(A-\lambda_{1}\right) u=0, B u=0\right\} \\
& \oplus\left\{\left(A-\lambda_{1}\right) u: u \in W^{2,2}(\Omega), B u=0\right\} .
\end{aligned}
$$


Indeed, it suffices to note that the operator $\mathfrak{A}$ is selfadjoint and that

$$
\operatorname{codim} R\left(\mathfrak{A}-\lambda_{1} I\right)=\operatorname{dim} N\left(\mathfrak{A}-\lambda_{1} I\right)=1 .
$$

Moreover, it follows from an application of the regularity theorem ([15, Theorem 8.2]) that

$$
N\left(\mathfrak{A}-\lambda_{1} I\right)=\left\{u \in C_{B}^{2+\alpha}(\bar{\Omega}):\left(A-\lambda_{1}\right) u=0 \text { in } \Omega\right\}=\operatorname{span}\left[\phi_{1}\right],
$$

and further that

$$
R\left(\mathfrak{A}-\lambda_{1} I\right) \cap C^{\alpha}(\bar{\Omega})=\left\{\left(A-\lambda_{1}\right) u: u \in C_{B}^{2+\alpha}(\bar{\Omega})\right\}
$$

where

$$
C_{B}^{2+\alpha}(\bar{\Omega})=\left\{u \in C^{2+\alpha}(\bar{\Omega}): B u=0\right\}
$$

Therefore, by restricting decomposition (3.1) to the subspace $Y=C^{\alpha}(\bar{\Omega})$ of $L^{2}(\Omega)$ we obtain the orthogonal decomposition

$$
\begin{aligned}
Y & =C^{\alpha}(\bar{\Omega}) \\
& =\left\{u \in C_{B}^{2+\alpha}(\bar{\Omega}):\left(A-\lambda_{1}\right) u=0 \text { in } \Omega\right\} \oplus\left\{\left(A-\lambda_{1}\right) u: u \in C_{B}^{2+\alpha}(\bar{\Omega})\right\} \\
& =\operatorname{span}\left[\phi_{1}\right] \oplus W,
\end{aligned}
$$

where

$$
W=\left\{\left(A-\lambda_{1}\right) u: u \in X\right\}, \quad X=C_{B}^{2+\alpha}(\bar{\Omega}) .
$$

If we define the orthogonal projection $Q$ from $X$ onto $W$ by the formula

$$
Q u=u-\left(\int_{\Omega} w(x) \phi_{1}(x) d x\right) \phi_{1} \quad \text { for all } u \in X,
$$

then it is easy to see that

$$
W=Q(X)=\left\{w \in Y: \int_{\Omega} w(x) \phi_{1}(x) d x=0\right\} .
$$

Moreover, by restricting decomposition (3.2) to the subspace $X=C_{B}^{2+\alpha}(\bar{\Omega})$ of $Y$ we obtain the orthogonal decomposition

$$
X=C_{B}^{2+\alpha}(\bar{\Omega})=\operatorname{span}\left[\phi_{1}\right] \oplus(W \cap X) .
$$

In other words, every function $u \in X$ can be written uniquely in the form

$$
u=t \phi_{1}+w(t), \quad t=\int_{\Omega} u(x) \phi_{1}(x) d x, \quad w(t) \in X \cap W .
$$

Then it is easy to verify that

$$
\begin{gathered}
\begin{cases}-A u+\lambda_{1} u+q(u)=h & \text { in } \Omega, \\
B u=0 & \text { in } \partial \Omega\end{cases} \\
\Longleftrightarrow\left\{\begin{array}{l}
u=t \phi_{1}+w(t), \\
-A w(t)+\lambda_{1} w(t)+Q\left(q\left(t \phi_{1}+w(t)\right)\right)=Q h \\
\int_{\Omega} Q\left(q\left(t \phi_{1}+w(t)\right)\right) \phi_{1}(x) d x=\int_{\Omega} h(x) \phi_{1}(x) d x .
\end{array}\right.
\end{gathered}
$$


Summing up, we are reduced to the infinite-dimensional equation

$$
-A w(t)+\lambda_{1} w(t)+Q\left(q\left(t \phi_{1}+w(t)\right)\right)=Q h \quad \text { in } \Omega
$$

and the one-dimensional system

$$
\int_{\Omega} Q\left(q\left(t \phi_{1}+w(t)\right)\right) \phi_{1}(x) d x=\int_{\Omega} h(x) \phi_{1}(x) d x .
$$

\subsection{Infinite-dimensional equation}

Now we consider the first (infinite-dimensional) equation (3.4), and formulate conditions which imply a priori estimates for solutions of equation (3.4) (Proposition 3.1), just as in the non-resonant case. To do this, we introduce a nonlinear map

$$
\Phi: \mathbf{R} \times(X \cap W) \longrightarrow W
$$

as follows:

$$
\Phi(t, w)=-A w+\lambda_{1} w+Q\left(q\left(t \phi_{1}+w\right)\right) \quad \text { for all }(t, w) \in \mathbf{R} \times(X \cap W) .
$$

Then it is easy to see that $\Phi \in C^{1}(\mathbf{R} \times(X \cap W), W)$.

The next proposition plays an essential role in the Lyapunov-Schmidt procedure:

Proposition 3.1 Assume that the nonlinear term $q(\xi)$ satisfies conditions (B.1) and (B.2). Then, for each function $h \in C^{\alpha}(\bar{\Omega})$ there exists a unique function $w(t)=$ $w(t, Q h) \in W \cap X$ which satisfies the following three conditions:

(i) $\Phi(t, w(t))=Q h$.

(ii) The function $t \mapsto w(t)$ is of class $C^{1}$.

(iii) The functions $w(t)$ are uniformly bounded in the space $C^{1+\alpha}(\bar{\Omega})$.

Proof (I) In order to prove assertions (i) and (ii), we shall apply the global inversion theorem (Theorem 2.3) to the map $\Phi(t, \cdot)$ for each $t \in \mathbf{R}$. The proof is divided into three steps.

Step 1: First, we show that the map

$$
\Phi(t, \cdot): X \cap W \longrightarrow W
$$

is proper for each $t \in \mathbf{R}$. The proof is divided into three steps.

Step 1-1: The next lemma proves the boundedness of the inverse $\left(-A+\lambda_{1} I\right)^{-1}$ on the space $W \cap X$ :

Lemma 3.1 There exists a positive constant $C\left(\lambda_{1}\right)$ depending on $\lambda_{1}$ such that

$$
\|u\|_{X} \leq C\left(\lambda_{1}\right)\left\|\left(-A+\lambda_{1}\right) u\right\|_{Y} \quad \text { for all } u \in W \cap X .
$$

Proof Assume, to the contrary, that, for every $n \in \mathbf{N}$ there is a function $u_{n} \in W \cap X$ such that

$$
\left\|u_{n}\right\|_{X}>n\left\|\left(-A+\lambda_{1}\right) u_{n}\right\|_{Y}
$$

If we let

$$
v_{n}=\frac{u_{n}}{\left\|u_{n}\right\|_{X}} \in X \cap W
$$


then we obtain from inequality (3.7) that

$$
\begin{aligned}
& \left\|v_{n}\right\|_{X}=1 \\
& \left\|\left(-A+\lambda_{1}\right) v_{n}\right\|_{Y}<\frac{1}{n} .
\end{aligned}
$$

However, it follows from an application of the Ascoli-Arzelà theorem that the injection $C^{2+\alpha}(\bar{\Omega}) \rightarrow C^{2}(\bar{\Omega})$ is compact. By assertion (3.8), we may assume that the sequence $\left\{v_{n}\right\}$ itself converges to some function $v^{*}$ in $C^{2}(\bar{\Omega})$ as $n \rightarrow \infty$ :

$$
v_{n} \longrightarrow v^{*} \text { in } C^{2}(\bar{\Omega}) \text { as } n \rightarrow \infty .
$$

We remark that the limit function $v^{*}(x)$ satisfies the boundary condition

$$
B v^{*}=\lim _{n \rightarrow \infty} B v_{n}=0 \quad \text { on } \partial \Omega .
$$

Furthermore, it follows from inequality (3.9) that

$$
\left(-A+\lambda_{1}\right) v_{n} \longrightarrow 0 \quad \text { in } C(\bar{\Omega}) \text { as } n \rightarrow \infty .
$$

Hence we have, by assertion (3.10),

$$
\left(-A+\lambda_{1}\right) v^{*}=\lim _{n \rightarrow \infty}\left(-A+\lambda_{1}\right) v_{n}=0 \quad \text { in } C(\bar{\Omega}) .
$$

By combining assertions (3.11) and (3.12), we obtain that

$$
\begin{cases}v^{*} \in C^{2}(\bar{\Omega}), & \\ \left(-A+\lambda_{1}\right) v^{*}=0 & \text { in } \Omega, \\ B v^{*}=0 & \text { on } \partial \Omega .\end{cases}
$$

Hence, it follows from an application of the regularity theorem for problem (3.13) ([15, Theorem 8.2]) that

$$
v^{*} \in C^{2+\alpha}(\bar{\Omega})
$$

so that

$$
v^{*} \in X=C_{B}^{2+\alpha}(\bar{\Omega}) .
$$

On the other hand, by applying [15, Theorem 1.2] with $\varphi:=0$ we can find a positive constant $C$ such that

$$
\|u\|_{X} \leq C\|A u\|_{Y} \quad \text { for all } u \in X=C_{B}^{2+\alpha}(\bar{\Omega}) .
$$

By using this inequality with $u:=v_{n}-v^{*}$, we obtain from assertions (3.8), (3.9), (3.10) and (3.12) that

$$
\begin{aligned}
\left\|v_{n}-v^{*}\right\|_{X} \leq & C\left\|A\left(v_{n}-v^{*}\right)\right\|_{Y} \\
= & C\left\|\left(A-\lambda_{1}\right)\left(v_{n}-v^{*}\right)+\lambda_{1}\left(v_{n}-v^{*}\right)\right\|_{Y} \\
\leq & C\left\|\left(A-\lambda_{1}\right) v_{n}\right\|_{Y}+C\left\|\left(A-\lambda_{1}\right) v^{*}\right\|_{Y} \\
& +C \lambda_{1}\left\|v_{n}-v^{*}\right\|_{Y} \\
\leq & \frac{C}{n}+C \lambda_{1}\left\|v_{n}-v^{*}\right\|_{Y} \longrightarrow 0 \quad \text { as } n \rightarrow \infty
\end{aligned}
$$


Hence we have, by assertion (3.8),

$$
\left\|v^{*}\right\|_{X}=\lim _{n \rightarrow \infty}\left\|v_{n}\right\|_{X}=1
$$

By using assertions (3.13) and (3.14), we can write the function $v^{*}(x)$ in the form

$$
v^{*}(x)=t_{0} \phi_{1}(x) \quad \text { for some } t_{0} \neq 0 \text {. }
$$

However, since the sequence $\left\{v_{n}\right\}$ in $X \cap W$ converges to the function $v^{*}(x)$ in $C^{2+\alpha}(\bar{\Omega})$ as $n \rightarrow \infty$, it follows that

$$
v^{*} \in X \cap W .
$$

In view of decomposition (3.3), this implies that

$$
v^{*}(x) \equiv 0 \quad \text { in } \Omega
$$

that is, $t_{0}=0$. This is a contradiction.

The proof of Lemma 3.1 is complete.

Step 1-2: Let $\left\{h_{n}\right\}$ be an arbitrary bounded sequence in $W$ such that

$$
\Phi\left(t, w_{n}\right)=h_{n}
$$

with $w_{n} \in X \cap W$, that is,

$$
\Phi\left(t, w_{n}\right)=\left(-A+\lambda_{1}\right) w_{n}+Q\left(q\left(t \phi_{1}+w_{n}\right)\right)=h_{n} .
$$

Then we show that the sequence $\left\{w_{n}\right\}$ is bounded in $X=C_{B}^{2+\alpha}(\bar{\Omega})$.

To do this, we rewrite equation (3.15) in the form

$$
\left(-A+\lambda_{1}\right) w_{n}=h_{n}-Q\left(q\left(t \phi_{1}+w_{n}\right)\right) .
$$

By conditions (B.1) and (B.2), we find that the sequence $\left\{q\left(t \phi_{1}+w_{n}\right)\right\}$ is bounded in the space $Y=C^{\alpha}(\bar{\Omega})$. Since the projection $Q: Y \rightarrow Y$ is bounded, it follows that the sequence

$$
\left\{Q\left(q\left(t \phi_{1}+w_{n}\right)\right)\right\}
$$

is bounded in the space $Y$. Therefore, by using inequality (3.6) with $u:=w_{n} \in X \cap W$ we obtain from equation (3.16) that the sequence $\left\{w_{n}\right\}$ is bounded in the space $X$.

Step 1-3: We show that if $\left\{w_{n}\right\}$ is a sequence in $X$ such that the sequence

$$
\left\{h_{n}\right\}=\left\{\Phi\left(t, w_{n}\right)\right\}
$$

converges to some function $h$ in $Y$ as $n \rightarrow \infty$, then the sequence $\left\{w_{n}\right\}$ contains a convergent subsequence in $X$. This proves that the mapping $\Phi(t, \cdot): X \cap W \rightarrow W$ is proper for each $t \in \mathbf{R}$.

First, we rewrite equation (3.17) as follows:

$$
A w_{n}=\lambda_{1} w_{n}-h_{n}+Q\left(q\left(t \phi_{1}+w_{n}\right)\right) .
$$

However, in Step 1-2 we have proved that the sequence

$$
\left\{\lambda_{1} w_{n}-h_{n}+Q\left(q\left(t \phi_{1}+w_{n}\right)\right)\right\}=\left\{A w_{n}\right\}
$$


is bounded in the space in $Y=C^{\alpha}(\bar{\Omega})$. Therefore, by applying [15, Theorem 1.2] with $\varphi:=0$ we obtain that the sequence $\left\{w_{n}\right\}$ is bounded in $X=C_{B}^{2+\alpha}(\bar{\Omega})$. Namely, we have, for some positive constant $c_{1}$,

$$
\left\|w_{n}\right\|_{C^{2+\alpha}(\bar{\Omega})} \leq c_{1}
$$

By the Ascoli-Arzelà theorem, we may assume that the sequence $\left\{w_{n}\right\}$ itself converges to some function $w^{*}$ in the space $C^{2}(\bar{\Omega})$ as $n \rightarrow \infty$ :

$$
w_{n} \longrightarrow w^{*} \text { in } C^{2}(\bar{\Omega}) \text { as } n \rightarrow \infty .
$$

Furthermore, it follows that

$$
\begin{aligned}
& \lambda_{1} w_{n}-h_{n}+Q\left(q\left(t \phi_{1}+w_{n}\right)\right) \\
& \longrightarrow \lambda_{1} w^{*}-h+Q\left(q\left(t \phi_{1}+w^{*}\right)\right) \quad \text { in } Y=C^{\alpha}(\bar{\Omega}) \text { as } n \rightarrow \infty
\end{aligned}
$$

Hence, by applying again $[15$, Theorem 1.2$]$ with $\varphi:=0$ we obtain from equation (3.18) that

$$
\begin{aligned}
& w_{n}=A^{-1}\left(\lambda_{1} w_{n}-h_{n}+Q\left(q\left(t \phi_{1}+w_{n}\right)\right)\right) \\
& \longrightarrow A^{-1}\left(\lambda_{1} w^{*}-h+Q\left(q\left(t \phi_{1}+w^{*}\right)\right)\right) \quad \text { in } X=C_{B}^{2+\alpha}(\bar{\Omega}) \text { as } n \rightarrow \infty .
\end{aligned}
$$

By combining assertions (3.19) and (3.20), we have proved that

$$
w^{*}=A^{-1}\left(\lambda_{1} w^{*}-h+Q\left(q\left(t \phi_{1}+w^{*}\right)\right)\right) \in X
$$

and further that

$$
w_{n} \longrightarrow w^{*} \text { in } X \text { as } n \rightarrow \infty \text {. }
$$

Step 2: Secondly, we prove that the map $\Phi(t, \cdot): X \cap W \rightarrow W$ is locally invertible on all of $W \cap X$, for each $t \in \mathbf{R}$.

We shall apply the local inversion theorem (Theorem 2.1) to the map $\Phi(t, \cdot)$. To do this, we have only to show that the Fréchet partial derivative

$$
\Phi_{w}(t, w) z=-A z+\lambda_{1} z+Q\left(q^{\prime}\left(t \phi_{1}+w\right) z\right), \quad z \in X \cap W,
$$

is invertible at each point $w \in X \cap W$.

The next lemma proves the Fredholm alternative theorem for the Fréchet partial derivative $\Phi_{w}(t, w)$ :

Lemma 3.2 The index of $\Phi_{w}(t, w): X \rightarrow Y$ is equal to zero:

$$
\operatorname{ind}\left(\Phi_{w}(t, w)\right)=\operatorname{dim} N\left(\Phi_{w}(t, w)\right)-\operatorname{codim} R\left(\Phi_{w}(t, w)\right)=0 .
$$

Proof If we associate with the linear elliptic boundary value problem

$$
\begin{cases}A v=f & \text { in } \Omega, \\ B v=0 & \text { on } \partial \Omega\end{cases}
$$

a continuous linear operator $\mathcal{A}$ by the formula

$$
\mathcal{A}=A: X \longrightarrow Y
$$


then it follows from an application of [15, Theorem 9.1] with $\varphi:=0$ that the operator $\mathcal{A}$ is a Fredholm operator with index zero:

$$
\text { ind } \mathcal{A}=\operatorname{dim} N(\mathcal{A})-\operatorname{codim} R(\mathcal{A})=0 .
$$

Moreover, if we let

$$
m(x)=q^{\prime}\left(t \phi_{1}(x)+w(x)\right) \quad \text { for all } x \in \bar{\Omega},
$$

then we obtain from condition (B.2) that

$$
m \in C^{\alpha}(\bar{\Omega}),
$$

and further that

$$
\begin{aligned}
Q\left(q^{\prime}\left(t \phi_{1}+w\right) z\right) & =Q(m(x) z) \\
& =m(x) z-\left(\int_{\Omega} m(y) z(y) \phi_{1}(y) d y\right) \phi_{1}(x) .
\end{aligned}
$$

By the Ascoli-Arzelà theorem, it follows that the operator $Q\left(q^{\prime}\left(t \phi_{1}+w\right) z\right): X \rightarrow Y$ is compact. Therefore, we find that the operator

$$
\Phi_{w}(t, w)=-\mathcal{A}+\lambda_{1} I+Q\left(q^{\prime}\left(t \phi_{1}+w\right) \cdot\right): X \longrightarrow Y
$$

is a Fredholm operator with index zero, since we have, by assertion (3.21),

$$
\text { ind }\left(\Phi_{w}(t, w)\right)=\operatorname{ind}(-\mathcal{A})=0 \text {. }
$$

The proof of Lemma 3.2 is complete.

By Lemma 3.2, it follows that $\Phi_{w}(t, w)$ is surjective if and only if it is injective. Rephrased, we find that $\Phi_{w}(t, w)$ is invertible if and only if the linear elliptic boundary value problem

$$
\begin{cases}-A z+\lambda_{1} z+Q\left(q^{\prime}\left(t \phi_{1}+w\right) z\right)=0 & \text { in } \Omega, \\ B z=0 & \text { on } \partial \Omega\end{cases}
$$

has only the trivial solution. However, by using formula (3.22) we can write problem (3.23) in the operator equation form

$$
-A z+\left(\lambda_{1}+m(x)\right) z-\left(\int_{\Omega} m(y) z(y) \phi_{1}(y) d y\right) \phi_{1}=0, \quad z \in W \cap X .
$$

Hence, we have, by equation (3.24),

$$
\begin{aligned}
0= & \int_{\Omega}\left(-A z(x)+\left(\lambda_{1}+m(x)\right) z(x)\right) \cdot z(x) d x \\
& -\left(\int_{\Omega} m(y) z(y) \phi_{1}(y) d y\right) \int_{\Omega} \phi_{1}(x) \cdot z(x) d x \\
= & -\int_{\Omega} A z(x) \cdot z(x) d x+\lambda_{1} \int_{\Omega} z(x)^{2} d x+\int_{\Omega} m(x) z(x)^{2} d x .
\end{aligned}
$$

On the other hand, by the variational formula it follows that

$$
\int_{\Omega} A z(x) \cdot z(x) d x \geq \lambda_{2} \int_{\Omega} z(x)^{2} d x
$$


since the function $z \in W \cap X$ is orthogonal to the eigenfunction $\phi_{1}(x)$ corresponding the first eigenvalue $\lambda_{1}$.

Therefore, by combining formula (3.25) and inequality (3.26) we obtain that

$$
\int_{\Omega}\left(\lambda_{1}+m(x)\right) z(x)^{2} d x=\int_{\Omega} A z(x) \cdot z(x) d x \geq \lambda_{2} \int_{\Omega} z(x)^{2} d x
$$

so that

$$
\int_{\Omega}\left(\lambda_{1}-\lambda_{2}+m(x)\right) z(x)^{2} d x \geq 0 .
$$

However, we have, by inequality (1.5),

$$
m(x)=q^{\prime}\left(t \phi_{1}(x)+w(x)\right)<\lambda_{2}-\lambda_{1} \quad \text { for all } x \in \bar{\Omega},
$$

and so

$$
\int_{\Omega}\left(\lambda_{1}-\lambda_{2}+m(x)\right) z(x)^{2} d x<0
$$

provided that $z(x) \not \equiv 0$ in $\Omega$.

By combining inequalities (3.27) and (3.28), we have proved that $z(x) \equiv 0$ in $\Omega$, that is, problem (3.23) has only the trivial solution.

Step 3: Thirdly, by Steps 1 and 2 we can apply the global inversion theorem (Theorem 2.3) to obtain that, for each $t \in \mathbf{R}$, the map

$$
\Phi(t, \cdot): X \cap W \longrightarrow W
$$

is a topological homeomorphism. Namely, for each $h \in C^{\alpha}(\bar{\Omega})$ there exists a unique function $w(t) \in X \cap W$ such that $\Phi(t, w(t))=Q h$. This proves the desired assertion (i).

Moreover, since the function

$$
\Phi(t, w(t))=-A w+\lambda_{1} w+Q\left(q\left(t \phi_{1}+w\right)\right)
$$

is of class $C^{1}$ with respect to $t$, it follows from an application of the implicit function theorem (Theorem 2.2) that the function $t \mapsto w(t)$ is of class $C^{1}$. This proves the desired assertion (ii).

(II) Finally, we prove assertion (iii). Since we have the formula

$$
Q h=\Phi(t, w(t))=-A w(t)+\lambda_{1} w(t)+Q\left(q\left(t \phi_{1}+w(t)\right)\right),
$$

it follows that

$$
\left\{\begin{array}{l}
u=t \phi_{1}+w(t), \\
\left(A-\lambda_{1}\right) w(t)=Q\left(q\left(t \phi_{1}+w(t)\right)\right)-Q h \in W, \\
w(t) \in X \cap W .
\end{array}\right.
$$

However, we find from inequality (3.6) that the operator

$$
A-\lambda_{1} I: X \cap W \longrightarrow W
$$

is an algebraic and topological isomorphism. If we let

$$
U(t)=Q\left(q\left(t \phi_{1}+w(t)\right)\right)-Q h,
$$

then we have the formula

$$
w(t)=\left(A-\lambda_{1} I\right)^{-1} U(t),
$$


and also, for some positive constant $c_{2}$,

$$
\begin{aligned}
\|U(t)\|_{C(\bar{\Omega})} & \leq\|Q h\|_{C(\bar{\Omega})}+\left\|Q\left(q\left(t \phi_{1}+w(t)\right)\right)\right\|_{C(\bar{\Omega})} \\
& \leq\|Q h\|_{C(\bar{\Omega})}+\sup _{\mathbf{R}}|q|+c_{2}\left\|\phi_{1}\right\|_{C(\bar{\Omega})} .
\end{aligned}
$$

For example, we may take

$$
c_{2}=\sup _{\mathbf{R}}|q| \cdot \int_{\Omega} \phi_{1}(x) d x
$$

This proves that the functions $U(t)$ are uniformly bounded in the space $C(\bar{\Omega})$. By applying [15, Theorem 1.1] for $p>N /(1-\alpha)$, we obtain from formula (3.29) that the functions $w(t)$ are uniformly bounded in the space $C^{1+\alpha}(\bar{\Omega})$. Indeed, it suffices to note that we have, by Sobolev's imbedding theorem (see [1, Theorem 4.12, Part II]),

$$
W^{2, p}(\Omega) \subset C^{2-N / p}(\bar{\Omega}) \subset C^{1+\alpha}(\bar{\Omega}),
$$

for $p>N /(1-\alpha)$.

Summing up, we have proved that, for some positive constant $c_{3}$,

$$
\|w(t)\|_{C^{1+\alpha}(\bar{\Omega})} \leq c_{3} .
$$

Now the proof of Proposition 3.1 is complete.

\section{Proof of Theorem 1.1}

This section is devoted to the proof of Theorem 1.1. Our proof of Theorem 1.1 is based on the Lyapunov-Schmidt procedure. More precisely, we study the one-dimensional system (3.5), and show that the behavior of the function

$$
\Gamma(t)=\int_{\Omega} q\left(t \phi_{1}(x)+w(t, x)\right) \phi_{1}(x) d x
$$

as $t \rightarrow \pm \infty$ is closely related to that of $q(\xi)$ as $\xi \rightarrow \pm \infty$. In fact, we show that

$$
\lim _{t \rightarrow \pm \infty} \Gamma(t)=q^{ \pm} \int_{\Omega} \phi_{1}(x) d x=\lim _{\xi \rightarrow \pm \infty} q(\xi) \cdot \int_{\Omega} \phi_{1}(x) d x .
$$

The proof is divided into two steps.

Step 1: First, by Subsection 3.1 we have the equivalent assertions

$$
\begin{gathered}
\\
\begin{cases}-A u+\lambda_{1} u+q(u)=h & \text { in } \Omega, \\
B u=0 & \text { on } \partial \Omega\end{cases} \\
\Longleftrightarrow\left\{\begin{array}{l}
u=t \phi_{1}+w(t), \\
-A w(t)+\lambda_{1} w(t)+Q\left(q\left(t \phi_{1}+w(t)\right)=Q h,\right. \\
\int_{\Omega} q\left(t \phi_{1}+w(t)\right) \phi_{1} d x=\int_{\Omega} h \phi_{1} d x
\end{array}\right. \\
\Longleftrightarrow\left\{\begin{array}{l}
u=t \phi_{1}+w(t), \\
-A w(t)+\lambda_{1} w(t)+Q\left(q\left(t \phi_{1}+w(t)\right)=Q h,\right. \\
\Gamma(t)=\int_{\Omega} h \phi_{1} d x .
\end{array}\right.
\end{gathered}
$$


Therefore, if we can find a point $t^{*} \in \mathbf{R}$ such that

$$
\Gamma\left(t^{*}\right)=\int_{\Omega} h(x) \phi_{1}(x) d x,
$$

then it follows that the function $u^{*}(x)$, defined by the formula

$$
u^{*}(x)=t^{*} \phi_{1}(x)+w\left(t^{*}\right)
$$

is a solution of problem (1.3).

Step 2: Under condition (1.6), we have only to show that there exists a point $t^{*} \in \mathbf{R}$ such that

$$
\Gamma\left(t^{*}\right)=\int_{\Omega} h(x) \phi_{1}(x) d x .
$$

To do this, let $\left\{t_{n}\right\}$ be an arbitrary sequence such that $t_{n} \rightarrow+\infty$, and let

$$
\begin{aligned}
& w_{n}(t)=w_{n}(t, x)=w\left(t_{n}, x\right), \\
& u_{n}(t)=u_{n}(t, x)=t_{n} \phi_{1}(x)+w_{n}(t, x) .
\end{aligned}
$$

By using Proposition 3.1, we may assume that the sequence $\left\{w_{n}\right\}$ itself converges to some function $w^{*}$ in the space $C^{1}(\bar{\Omega})$ as $n \rightarrow \infty$. Then we have, for some positive constant $c$,

$$
\begin{aligned}
\left|u_{n}(t, x)\right|=\left|t_{n} \phi_{1}(x)+w_{n}(t, x)\right| & \geq\left|t_{n}\right| \phi_{1}(x)-\left|w_{n}(t, x)\right| \\
& \geq\left|t_{n}\right| \phi_{1}(x)-c \text { for all } x \in \Omega .
\end{aligned}
$$

This proves that, as $n \rightarrow \infty$,

$$
\left|u_{n}(t, x)\right| \longrightarrow+\infty \quad \text { in } \Omega \text { as } n \rightarrow \infty,
$$

since $\phi_{1}(x)>0$ in $\Omega$. Hence it follows from an application of Lebesgue's bounded convergence theorem that we have, by condition (B.3),

$$
\begin{aligned}
\lim _{n \rightarrow \infty} \Gamma\left(t_{n}\right) & =\lim _{t_{n} \rightarrow+\infty} \int_{\Omega} q\left(u_{n}(t, x)\right) \phi_{1}(x) d x \\
& =\int_{\Omega} \lim _{n \rightarrow \infty} q\left(u_{n}(t, x)\right) \phi_{1}(x) d x \\
& =q^{+} \int_{\Omega} \phi_{1}(x) d x .
\end{aligned}
$$

This proves that

$$
\lim _{t \rightarrow+\infty} \Gamma(t)=q^{+} \int_{\Omega} \phi_{1}(x) d x
$$

since the sequence $\left\{t_{n}\right\}$ is arbitrary.

Similarly, we can prove that

$$
\lim _{t \rightarrow-\infty} \Gamma(t)=q^{-} \int_{\Omega} \phi_{1}(x) d x .
$$

Therefore, the desired assertion (4.2) follows from an application of the intermediate value theorem, since the function $\Gamma(t)$ is continuous and since condition (1.6) is satisfied.

The proof of Theorem 1.1 is complete. 


\section{Proof of Theorem 1.2}

In this section we prove Theorem 1.2. To do this, we study the one-dimensional system (3.5), and show that the behavior of the function $t \Gamma(t)$ as $t \rightarrow \pm \infty$ is closely related to that of $\xi q(\xi)$ as $\xi \rightarrow \pm \infty$, where the function $\Gamma(t)$ is defined by formula (4.1). In fact, we show that

$$
\lim _{t \rightarrow \pm \infty} t \Gamma(t)=\sigma|\Omega|=\lim _{\xi \rightarrow \pm \infty} \xi q(\xi) \cdot|\Omega|
$$

where $|\Omega|$ is the volume of $\Omega$. The proof is divided into two steps.

Step 1: First, under condition (1.7) we have the equivalent assertions

$$
\begin{aligned}
& \begin{cases}-A u+\lambda_{1} u+q(u)=h & \text { in } \Omega, \\
B u=0 & \text { on } \partial \Omega\end{cases} \\
\Longleftrightarrow & \left\{\begin{array}{l}
u=t \phi_{1}+w(t), \\
-A w(t)+\lambda_{1} w(t)+Q\left(q\left(t \phi_{1}+w(t)\right)=Q h,\right. \\
\Gamma(t)=0 .
\end{array}\right.
\end{aligned}
$$

Therefore, if we can find a point $t^{*} \in \mathbf{R}$ such that

$$
\Gamma\left(t^{*}\right)=0
$$

then it follows that the function $u^{*}(x)$, defined by the formula

$$
u^{*}(x)=t^{*} \phi_{1}(x)+w\left(t^{*}\right)
$$

is a solution of problem (1.3).

Step 2: Secondly, we show that there exists a point $t^{*} \in \mathbf{R}$ such that

$$
\Gamma\left(t^{*}\right)=\int_{\Omega} h(x) \phi_{1}(x) d x=0 .
$$

To do this, we consider the function

$$
t \Gamma(t)=\int_{\Omega} q\left(t \phi_{1}+w(t)\right)\left(t \phi_{1}+w(t)\right) d x-\int_{\Omega} q\left(t \phi_{1}+w(t)\right) w(t) d x .
$$

Then it suffices to prove that

$$
\lim _{t \rightarrow \pm \infty} t \Gamma(t)=\sigma|\Omega| .
$$

Indeed, the desired assertion (5.1) follows from an application of the intermediate value theorem, since the function $\Gamma(t)$ is continuous.

Now, let $\left\{t_{n}\right\}$ be an arbitrary sequence such that $\left|t_{n}\right| \rightarrow \infty$, and let

$$
\begin{aligned}
& w_{n}(t)=w_{n}(t, x)=w\left(t_{n}, x\right), \\
& u_{n}(t)=u_{n}(t, x)=t_{n} \phi_{1}(x)+w_{n}(t, x) .
\end{aligned}
$$

Then it follows from equation (5.2) that

$$
t_{n} \Gamma\left(t_{n}\right)=\int_{\Omega} q\left(u_{n}(t, x)\right) u_{n}(t, x) d x-\int_{\Omega} q\left(u_{n}(t, x)\right) w_{n}(t, x) d x .
$$


By virtue of assertion (iii) of Proposition 3.1 with $w(t):=w_{n}(t, x)$, we may assume that the sequence $\left\{w_{n}\right\}$ itself converges to some function $w^{*}$ in the space $C^{1}(\bar{\Omega})$ as $n \rightarrow \infty$. Then we have, for some positive constant $c$,

$$
\begin{aligned}
\left|u_{n}(t, x)\right|=\left|t_{n} \phi_{1}(x)+w_{n}(t, x)\right| & \geq\left|t_{n}\right| \phi_{1}(x)-\left|w_{n}(t, x)\right| \\
& \geq\left|t_{n}\right| \phi_{1}(x)-c \text { for all } x \in \Omega .
\end{aligned}
$$

This proves that, as $\left|t_{n}\right| \rightarrow \infty$,

$$
\left|u_{n}(t, x)\right| \longrightarrow \infty \quad \text { in } \Omega \text { as } n \rightarrow \infty,
$$

since $\phi_{1}(x)>0$ in $\Omega$. Hence it follows from an application of Lebesgue's bounded convergence theorem that we have, by condition (B.4),

$$
\begin{aligned}
& \lim _{n \rightarrow \infty} \int_{\Omega} q\left(u_{n}(t, x)\right) u_{n}(t, x) d x=\sigma|\Omega|, \\
& \lim _{n \rightarrow \infty} \int_{\Omega} q\left(u_{n}(t, x)\right) w_{n}(t, x) d x=0 .
\end{aligned}
$$

Therefore, we obtain from formula (5.4) that

$$
\lim _{n \rightarrow \infty} t_{n} \Gamma\left(t_{n}\right)=\sigma|\Omega|
$$

This proves the desired assertion (5.3), since the sequence $\left\{t_{n}\right\}$ is arbitrary.

The proof of Theorem 1.2 is complete.

\section{Proof of Theorem 1.3}

This final section is devoted to the proof of Theorem 1.3. The "if" part is an immediate consequence of Theorem 1.1.

We have only to prove the "only if" part and the uniqueness result. Roughly speaking, we shall show that the behavior of $\Gamma(t)$ as $t \rightarrow \pm \infty$ is closely related to that of $q(\xi)$ as $\xi \rightarrow \pm \infty$. In fact, we show that the function $\Gamma(t)$ is strictly increasing on $\mathbf{R}$ (see Lemma 6.1). The proof is divided into three steps.

Step 1: First, we recall that

$$
\Gamma(t)=\int_{\Omega} q\left(t \phi_{1}(x)+w(t, x)\right) \phi_{1}(x) d x
$$

and that the function $w(t)=w(t, \cdot)$ is differentiable with respect to $t$. By differentiating the equation

$$
-A w(t)+\lambda_{1} w(t)+Q\left(q\left(t \phi_{1}+w(t)\right)\right)=Q h,
$$

we obtain that the derivative $w^{\prime}(t)=w_{t}(t, \cdot)$ satisfies the equation

$$
\begin{aligned}
& -A w^{\prime}(t)+\lambda_{1} w^{\prime}(t)+\frac{d}{d t}\left(Q\left(q\left(t \phi_{1}+w(t)\right)\right)\right) \\
= & -A w^{\prime}(t)+\lambda_{1} w^{\prime}(t)+Q\left(q^{\prime}\left(t \phi_{1}+w(t)\right)\left(\phi_{1}+w^{\prime}(t)\right)\right) \\
= & 0 .
\end{aligned}
$$


However, we find that the derivative $\Gamma^{\prime}(t)$ is given by the formula

$$
\Gamma^{\prime}(t)=\int_{\Omega} q^{\prime}\left(t \phi_{1}(x)+w(t, x)\right)\left(\phi_{1}(x)+w_{t}(t, x)\right) \phi_{1}(x) d x,
$$

so that

$$
\begin{aligned}
& Q\left(q^{\prime}\left(t \phi_{1}+w(t)\right)\left(\phi_{1}+w^{\prime}(t)\right)\right) \\
= & q^{\prime}\left(t \phi_{1}+w(t)\right)\left(\phi_{1}+w^{\prime}(t)\right) \\
& -\left(\int_{\Omega} q^{\prime}\left(t \phi_{1}(y)+w(t, y)\right)\left(\phi_{1}(y)+w_{t}(t, y)\right) \phi_{1}(y) d y\right) \phi_{1}(x) \\
= & q^{\prime}\left(t \phi_{1}+w(t)\right)\left(\phi_{1}+w^{\prime}(t)\right)-\Gamma^{\prime}(t) \phi_{1}(x) .
\end{aligned}
$$

Hence we can rewrite equation (6.1) in the form

$$
-A w^{\prime}(t)+\lambda_{1} w^{\prime}(t)+q^{\prime}\left(t \phi_{1}+w(t)\right)\left(\phi_{1}+w^{\prime}(t)\right)-\Gamma^{\prime}(t) \phi_{1}(x)=0 .
$$

Step 2: Now we can prove the following lemma:

Lemma 6.1 The function $\Gamma(t)$ is strictly increasing on $\mathbf{R}$, that is,

$$
\Gamma^{\prime}(t)>0 \quad \text { on } \mathbf{R} .
$$

Proof By assertions (4.3) and (4.4), we remark that

$$
\begin{aligned}
& q^{-} \int_{\Omega} \phi_{1}(x) d x<\Gamma(t)<q^{+} \int_{\Omega} \phi_{1}(x) d x, \\
& \lim _{t \rightarrow+\infty} \Gamma(t)=q^{+} \int_{\Omega} \phi_{1}(x) d x \\
& \lim _{t \rightarrow-\infty} \Gamma(t)=q^{-} \int_{\Omega} \phi_{1}(x) d x .
\end{aligned}
$$

Hence it suffices to show that

$$
\Gamma^{\prime}(t) \neq 0 \quad \text { on } \mathbf{R} .
$$

Assume, to the contrary, that there exists a point $t^{*} \in \mathbf{R}$ such that

$$
\Gamma^{\prime}\left(t^{*}\right)=0
$$

If we let

$$
\begin{aligned}
& u^{*}(x)=t^{*} \phi_{1}(x)+w\left(t^{*}\right)=t^{*} \phi_{1}(x)+w\left(t^{*}, x\right) \in X \\
& z^{*}(x)=\phi_{1}(x)+w^{\prime}\left(t^{*}\right)=\phi_{1}(x)+w_{t}\left(t^{*}, x\right) \in X
\end{aligned}
$$

then we have, by equation (6.3) with $t:=t^{*}$,

$$
\begin{aligned}
& -A z^{*}+\lambda_{1} z^{*}+q^{\prime}\left(u^{*}\right) z^{*} \\
= & -A w^{\prime}\left(t^{*}\right)+\lambda_{1} w^{\prime}\left(t^{*}\right)+q^{\prime}\left(t^{*} \phi_{1}+w\left(t^{*}\right)\right)\left(\phi_{1}+w^{\prime}\left(t^{*}\right)\right) \\
= & 0 \quad \text { in } \Omega .
\end{aligned}
$$

Moreover, we remark that

$$
\int_{\Omega} z^{*}(x) \cdot \phi_{1}(x) d x=\int_{\Omega}\left(\phi_{1}(x)+w_{t}\left(t^{*}, x\right)\right) \phi_{1}(x) d x=\int_{\Omega} \phi_{1}(x) \cdot \phi_{1}(x) d x=1,
$$


since the function $w_{t}\left(t^{*}, \cdot\right) \in W$ is orthogonal to the eigenfunction $\phi_{1}$. In particular, it follows that $z^{*}(x) \not \equiv 0$ in $\Omega$.

If we let

$$
m^{*}(x)=\lambda_{1}+q^{\prime}\left(u^{*}(x)\right)=\lambda_{1}+q^{\prime}\left(t^{*} \phi_{1}(x)+w\left(t^{*}, x\right)\right) \quad \text { for all } x \in \bar{\Omega},
$$

then it follows from condition (B.2) that $m^{*} \in C(\bar{\Omega})$. Therefore, we obtain from equation (6.4) that the non-trivial function $z^{*}(x)$ is a solution of the eigenvalue problem with the weight $m^{*}(x)$

$$
\begin{cases}A z^{*}=m^{*}(x) z^{*} & \text { in } \Omega, \\ B z^{*}=0 & \text { on } \partial \Omega .\end{cases}
$$

This proves that, for some $k \geq 1$,

$$
\lambda_{k}\left(m^{*}\right)=1
$$

We recall (see [17, Proposition 3.4]) that $\lambda_{k}\left(m^{*}\right)$ is the $\mathrm{k}$-th eigenvalue of the elliptic boundary value problem with the weight $m^{*}(x)$ :

$$
\begin{cases}A u=\lambda m^{*}(x) u & \text { in } \Omega \\ B u=0 & \text { on } \partial \Omega\end{cases}
$$

On the other hand, since we have, by inequality (1.5),

$$
m^{*}(x)=\lambda_{1}+q^{\prime}\left(u^{*}(x)\right)<\lambda_{2} \quad \text { for all } x \in \bar{\Omega},
$$

it follows from an application of the comparison property of eigenvalues ([17, Corollary 3.6]) that

$$
\lambda_{2}\left(m^{*}\right)>1
$$

Hence we have the assertion

$$
\lambda_{1}\left(m^{*}\right)=1 .
$$

By applying the Krein-Rutman theorem (see [16, Theorem 2.1]), we obtain that the corresponding eigenfunction $z^{*}(x)$ does not change sign in $\Omega$.

However, since $q^{\prime}(\xi)>0$ on $\mathbf{R}$ and since $\phi_{1}(x)>0$ in $\Omega$, we have, by formula (6.2) with $t:=t^{*}$,

$$
0=\Gamma^{\prime}\left(t^{*}\right)=\int_{\Omega} q^{\prime}\left(u^{*}(x)\right) \cdot z^{*}(x) \cdot \phi_{1}(x) d x \begin{cases}>0 & \text { if } z^{*}(x)>0 \text { in } \Omega \\ <0 & \text { if } z^{*}(x)<0 \text { in } \Omega\end{cases}
$$

This contradiction proves Lemma 6.1.

Step 3: Summing up, we have proved that problem (1.3) has a unique solution $u \in C^{2+\alpha}(\bar{\Omega})$ if and only if the function $h \in C^{\alpha}(\bar{\Omega})$ satisfies condition (1.6).

Now the proof of Theorem 1.3 is complete.

Acknowledgements This research is partially supported by Grant-in-Aid for General Scientific Research (No. 19540162), Ministry of Education, Culture, Sports, Science and Technology, Japan. 


\section{References}

1. Adams, R.A., Fournier, J.J.F.: Sobolev spaces, second edition. Academic Press, Amsterdam Heidelberg New York Oxford (2003)

2. Amann, H.: Fixed point equations and nonlinear eigenvalue problems in ordered Banach spaces. SIAM Rev. 18, 620-709 (1976)

3. Ambrosetti, A., Prodi, G.: A primer of nonlinear analysis. Cambridge University Press, Cambridge (1993)

4. Chang, K.C.: Methods in nonlinear analysis. Springer-Verlag, Berlin Heidelberg New York (2005)

5. de Figueiredo, D.G.: Positive solutions of semilinear elliptic problems. In: Differential equations, Lecture Notes in Mathematics, No. 957, pp. 34-87, Springer-Verlag, Berlin Heidelberg New York (1982)

6. Dieudonné, J.: Foundations of modern analysis. Academic Press, New York London (1969)

7. Hörmander, L.: The analysis of linear partial differential operators III. Springer-Verlag, Berlin Heidelberg New York Tokyo (1985)

8. Kreı̆n, M.G., Rutman, M.A.: Linear operators leaving invariant a cone in a Banach space. Amer. Math. Soc. Transl. 10, 199-325 (1962)

9. Landesman, E.A., Lazer, A.C.: Nonlinear perturbations of linear elliptic boundary value problems at resonance. J. Math. Mech. 19, 609-623 (1970)

10. Manes, A., Micheletti, A.M.: Un'estensione della teoria variazionale classica degli autovalori per operatori ellitici del secondo ordine. Boll. Un. Mat. Ital. 7, 285-301 (1973)

11. Marsden, J.E., Hughes, T.J.R.: Mathematical foundations of elasticity. Prentice-Hall, Inc., Englewood Cliffs, New Jersey (1983)

12. Nirenberg, L.: Topics in nonlinear functional analysis. Courant Institute of Mathematical Sciences, New York University, New York (1974)

13. Runst, T., Sickel, W.: Sobolev spaces of fractional order, Nemytskij operators, and nonlinear partial differential equations. Walter de Gruyter, Berlin New York (1996)

14. Taira, K.: Analytic semigroups and semilinear initial-boundary value problems. London Mathematical Society Lecture Note Series, Vol. 223. Cambridge University Press, Cambridge (1995)

15. Taira, K.: Semigroups, boundary value problems and Markov processes. Springer-Verlag, Berlin Heidelberg New York (2004)

16. Taira, K.: Degenerate elliptic boundary value problems with indefinite weights. Mediterranean J. Math. 5, 133-162 (2008)

17. Taira, K.: Degenerate elliptic boundary value problems with asymmetric nonlinearity. J. Math. Soc. Japan 62, 431-465 (2010)

18. Zeidler, E.: Nonlinear functional analysis and its applications I. Springer-Verlag, New York Berlin Heidelberg (1986) 